\title{
Fetal programming and epigenetic mechanisms in arterial hypertension
}

\author{
Urs Scherrer ${ }^{\mathrm{a}, \mathrm{b}, *}$, Stefano F. Rimoldi ${ }^{\mathrm{a}}$, Claudio Sartori ${ }^{\mathrm{c}}$, Franz H. Messerli, ${ }^{\mathrm{a}, \mathrm{d}}$, \\ and Emrush Rexhaj ${ }^{\mathrm{a}, *}$
}

\begin{abstract}
Purpose of review
To provide an overview of available evidence of the potential role of epigenetics in the pathogenesis of hypertension and vascular dysfunction.

\section{Recent findings}

Arterial hypertension is a highly heritable condition. Surprisingly, however, genetic variants only explain a tiny fraction of the phenotypic variation and the term 'missing heritability' has been coined to describe this phenomenon. Recent evidence suggests that phenotypic alteration that is unrelated to changes in DNA sequence (thereby escaping detection by classic genetic methodology) offers a potential explanation. Here, we present some basic information on epigenetics and review recent work consistent with the hypothesis of epigenetically induced arterial hypertension.
\end{abstract}

\section{Summary}

New technologies that enable the rigorous assessment of epigenetic changes and their phenotypic consequences may provide the basis for explaining the missing heritability of arterial hypertension and offer new possibilities for treatment and/or prevention.

\section{Keywords}

epigenetics, hypertension, in-vitro fertilization, preeclampsia, vascular dysfunction

\section{INTRODUCTION}

Hypertension is defined as a condition with a SBP at least $140 \mathrm{~mm} \mathrm{Hg}$ or a DBP at least $90 \mathrm{~mm} \mathrm{Hg}$, it affects more than 1 billion people worldwide, and its cost makes up an important part of the healthcare expenses in industrialized countries [1]. Hypertension is the most prevalent cardiovascular risk factor worldwide [2]. Although in approximately $10 \%$ of hypertensive patients an underlying cause can be identified, in the remaining 90\% the cause of high blood pressure remains unknown, and the term 'essential hypertension' has been coined to describe this condition. There is abundant evidence that essential hypertension is a highly heritable condition, and heritability of hypertension has been estimated at between 30 and 40\% [3,4]. In line with this concept, large genome-wide linkage and association studies have identified many novel genetic variants associated with increased arterial blood pressure, unmistakably demonstrating the multigenic complexity of essential hypertension. Surprisingly, however, these genetic variants, individually and collectively, only explain a tiny fraction of phenotypic variation and disease risk [1]. Of note, though, similar differences between estimated and observed variance were found in genome-wide association studies of other complex diseases in humans, and the term 'missing heritability' has been coined to describe this difference. Accordingly, in research on hypertension (and other complex diseases), the search for missing heritability has emerged as a major goal.

Although several possibilities such as unexplored portions of the genome, gene interactions, or untested genetic variants, to name just a few, have been suggested $[5,6]$, phenotypic alteration,

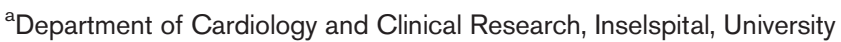
Hospital Bern, Bern, Switzerland, ${ }^{b}$ Facultad de Ciencias, Departamento de Biología, Universidad de Tarapacá, Arica, Chile, 'Department of Internal Medicine, University Hospital, Lausanne, Switzerland and ${ }^{d}$ Mount Sinai Health Medical Center, Icahn School of Medicine, Division of Cardiology, New York, USA

Correspondence to Urs Scherrer, MD, Department of Cardiology and Clinical Research Inselspital, University Hospital Bern, CH-3010 Bern, Switzerland. Tel: +4131632 01 02; fax: 413163242 11; e-mail: urs.scherrer2@insel.ch

*Emrush Rexhaj and Urs Scherrer contributed equally to the writing of this article.

Curr Opin Cardiol 2015, 30:393-397

DOI:10.1097/HCO.0000000000000192 


\section{KEY POINTS}

- Although a highly heritable trait, the pathogenesis of essential hypertension is poorly understood.

- Genetic variants account for only a small proportion of blood pressure variation.

- The concept of missed heritability is defined as the difference between estimated and observed variance.

- Fetal programming and epigenetic alterations may importantly contribute to explain this phenomenon.

which is unrelated to changes in DNA sequence and thereby is not detected using classic genetic methodology, offers an attractive hypothesis. In line with this hypothesis, there is emerging evidence demonstrating epigenetic alteration of genes affecting important pathways in blood pressure regulation $[7,8]$ that, if heritable, could provide the scientific basis explaining part of the missing heritability in essential hypertension. Here, we will briefly present some basic information on epigenetics and then review recent studies in experimental animal models and humans consistent with the concept of epigenetically induced arterial hypertension.

\section{FETAL PROGRAMMING OF ADULT DISEASE HYPOTHESIS}

Epidemiological data suggest that environmental factors acting during the fetal and perinatal period alter the regulation of cardiovascular and metabolic homeostasis, which predisposes to cardiovascular and/or metabolic disease later in life. These observations have led to the so-called 'fetal programming of adult disease hypothesis' or 'Barker hypothesis' [9]. This hypothesis suggests that pathological events during embryonic, fetal, and early postnatal life have persistent effects on cardiovascular and metabolic homeostasis, and thereby increase the risk for cardiovascular disease and diabetes later in life (Fig. 1). Although most evidence for fetal programming stems from epidemiological studies in humans and experimental work in mammals, there is also evidence for fetal programming and its intergenerational transmission in lower organisms, such as the fruit fly Drosophila melanogaster [10]. For example, in humans, maternal malnutrition during early gestation (Dutch famine studies) increases the prevalence of coronary heart disease in the offspring [11]. In line with these observations in humans, maternal diet restriction during pregnancy in rodents, an experimental model mimicking preeclampsia in humans, induces systemic and pulmonary hypertension in the offspring, which appears to be related to an epigenetic mechanism $[8,12]$.

\section{EPIGENETICS}

Epigenetics is defined as the transmission of gene expression patterns from one cell generation to the next, which do not rely on differences in DNA sequence. There exist many examples of cell functions (e.g., tissue specificity, X-chromatin inactivation, germline specificity) that are critically dependent on epigenetic control of gene expression. Technological progress that allows epigenetic profiling across the genome has allowed documenting in experimental animals that epigenetic modifications take place not only during early development but also later in life $[13,14]$. The term 'epigenetics' refers to chromatin-based mechanisms that regulate gene expression without affecting the DNA sequence per se. Although epigenetic changes may take place throughout life, the most important epigenetic modifications take place during embryonic, fetal, and early postnatal development. These epigenetic

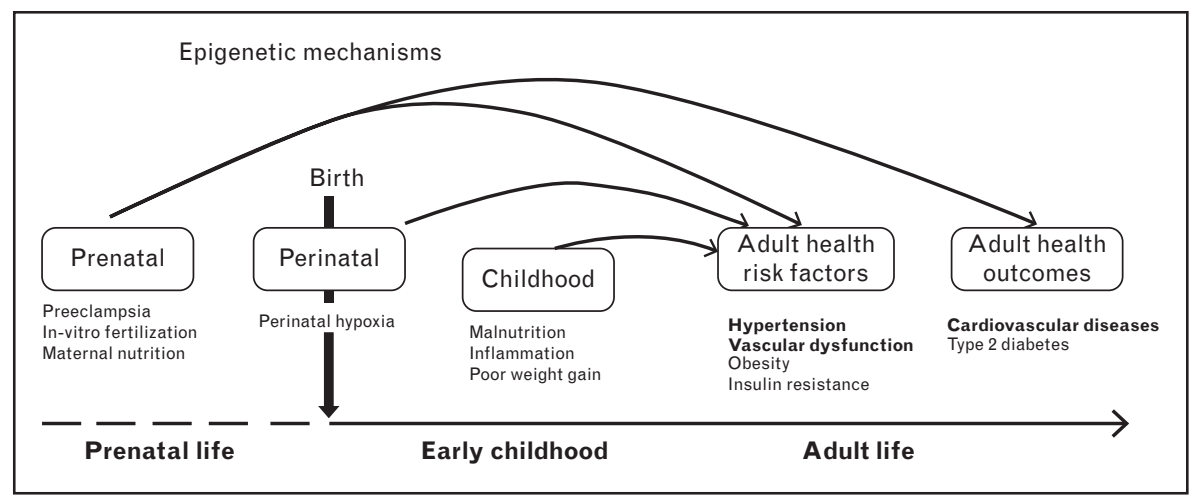

FIGURE 1. Fetal programming of arterial hypertension. Schematic representation of the effects of environmental factors occurring during early development on cardiovascular diseases later in life. The main putative prenatal, perinatal, and childhood factors that may lead to epigenetic modifications and in turn influence adult health outcomes are represented. 
mechanisms are essential for the adequate regulation of gene expression during these different phases of early life. The important activity of the epigenome during early life also renders it particularly vulnerable. In line with this concept, there is increasing data indicating that during early life, epigenetic mechanisms are strongly influenced by the environment [15"] and that environmentally induced epigenetic modifications are heritable throughout generations. The latter is related to the fact that epigenetic changes not only affect the phenotype of the immediate offspring, but, because some of these changes are not erased during gametogenesis, also affect the phenotype and health of future generations. It is remarkable that epigenetic inheritance of premature vascular ageing and arterial hypertension in mice generated by in-vitro fertilization appears as strong as conventional genetic effects observed in knockout animal models [8].

The best-studied epigenetic mechanisms involve DNA methylation and histone modifications (acetylation and deacetylation; Fig. 2). Other mechanisms, such as micro-RNAs, have also been described [1].

\section{ROLE OF EPIGENETICS IN HYPERTENSION}

\section{Arterial hypertension and premature vascular ageing induced by in-vitro fertilization}

Assisted reproductive technologies (ART), which initially were developed to treat women with tubal disease, have been applied to an ever expanding list of other situations, resulting in an exponential growth of the number of babies born by this procedure, who now account for $2-5 \%$ of births in developed countries [16]. Based on emerging data showing that the early embryo is particularly sensitive to environmental insults, we speculated that ART alters cardiovascular function in the offspring. In line with this speculation, we found that young, apparently healthy ART children display signs of premature vascular ageing of the systemic circulation together with pulmonary vascular dysfunction predisposing to exaggerated hypoxic pulmonary hypertension [17-20]. Most importantly, recent data indicate that ART-induced vascular dysfunction translates into increased arterial blood pressure. Ceelen et al. [21] reported higher systolic and diastolic office blood pressure in ART children than in spontaneously conceived control children, and preliminary data using 24-h ambulatory blood pressure measurements show that in ART children, vascular dysfunction is associated with increased SBP and DBP compared with control children [22]. These differences in blood pressure between ART and control children are clinically important, as childhood blood pressure tracks into later life [23]. Parenthetically, it is well known that arterial hypertension is often associated with insulin resistance [24], and defective nitric oxide-dependent insulin stimulation of blood flow and substrate delivery to skeletal muscle tissue, which leads to insulin resistance in experimental animal models and humans, may represent an underlying mechanism $[20,25,26]$.

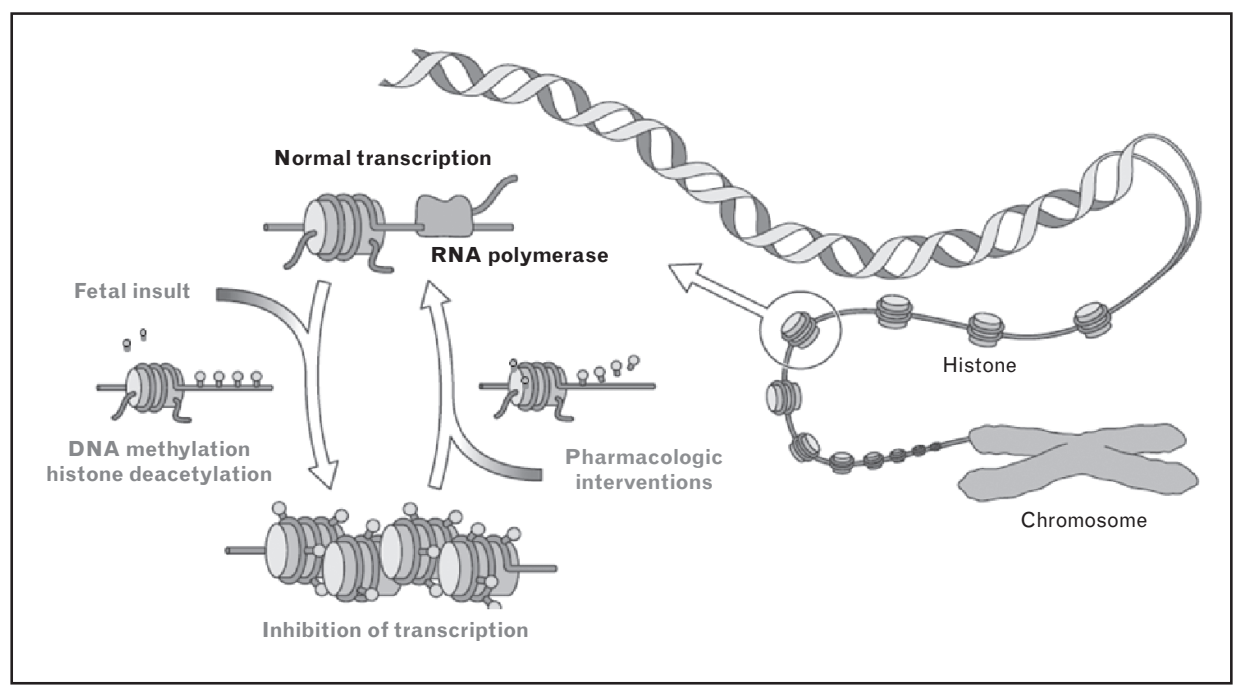

FIGURE 2. Epigenetic mechanisms of gene regulation. Fetal/perinatal insults induce epigenetic alterations, such as DNA methylation and histone deacetylation, that modify gene expression. DNA methylation occurs at cytosine-phosphatidyl-guanine $(C p G)$ dinucleotides and is responsible for inhibition of transcription. Hyperacetylation of the histones correlates with transcriptional activation, whereas deacetylation is associated with transcriptional inactivation. Epigenetic alterations can be reversed by pharmacological agents, such as histone deacetylase inhibitors. 
Accordingly, a recent study demonstrated insulin resistance in young adults conceived by ART [27], suggesting that ART-induced vascular dysfunction may facilitate diabetes later in life.

\section{Epigenetic mechanisms contribute to assisted reproductive technologies-induced arterial hypertension and premature vascular ageing}

Consistently with observations in humans, ART induces arterial hypertension and premature vascular ageing in mice $\left[8,28^{\prime \prime}\right]$. Most importantly, observations in ART mice provide direct evidence for the importance of ART-induced epigenetic alterations underpinning the cardiovascular alterations. The evidence is as follows. In ART mice, the methylation of the promoter of the gene coding for endothelial nitric oxide synthase (eNOS) is altered in the aorta. These epigenetic alterations in the vasculature have important consequences, as shown by decreased vascular eNOS and eNOS mRNA expression and lower nitric oxide plasma concentration in ART than in control mice [29]. The functional importance of these epigenetic alterations is further demonstrated by the effects of histone deacetylase inhibitor administration to ART mice. These drugs have been shown to reverse epigenetic and phenotypic changes induced by pathologic events during early life and to prevent transmission of these changes to the progeny $[29,30]$. Consistently with these observations, butyrate administration to adult male ART mice normalizes the methylation and expression of the eNOS gene in vascular tissue together with vascular responsiveness to acetylcholine and prevents the transmission of these alterations to the progeny $[29,30]$.

Collectively, these findings indicate that in mice, ART alters the cardiovascular phenotype by an epigenetic mechanism, which changes the entire chain of events starting from altered eNOS methylation in the vasculature over endothelial dysfunction and premature vascular senescence to arterial hypertension and possibly premature mortality [29]. It is tempting to speculate that ART-induced epigenetic alterations also play a role in ART-induced premature vascular ageing and arterial hypertension in humans.

\section{Arterial hypertension and premature vascular ageing in offspring of preeclampsia}

Preeclampsia is characterized by hypertension, proteinuria, and edema, and complicates up to $10 \%$ of pregnancies in Western countries. Recent data in young, apparently healthy offspring of preeclampsia demonstrate premature vascular ageing of the systemic circulation and exaggerated hypoxic pulmonary vasoconstriction that are related to preeclampsia per se rather than to a genetic abnormality that predisposes the mother to preeclampsia and the offspring to vascular dysfunction $[31,32]$. These vascular alterations are clinically important, because they are associated with a marked increase of the risk for stroke later in life [33].

Epigenetic mechanism. To test for underlying mechanisms, we studied offspring of restrictive diet pregnancy (RDP) in mice, a mouse model mimicking several aspects of preeclampsia in humans [34]. We found that in addition to systemic vascular dysfunction and arterial hypertension [22], endothelium-dependent pulmonary artery vasodilation in vitro was impaired, and hypoxia-induced pulmonary hypertension and right ventricular hypertrophy in vivo were exaggerated in offspring of $\mathrm{RDP}$ compared with control mice. Most importantly, pulmonary vascular dysfunction was associated with altered lung DNA methylation. Administration of histone deacetylase inhibitors to offspring of RDP normalized pulmonary DNA methylation and pulmonary vascular function, demonstrating the importance of epigenetic alterations in causing vascular dysfunction in this model. We speculate that epigenetic alterations also underpin vascular dysfunction in offspring of preeclampsia in humans.

\section{CONCLUSION}

Essential hypertension still remains poorly understood. Hypertension is a highly heritable trait, but discovered genetic variants account for only a very small fraction of phenotypic variation. Missing heritability, which is the difference between estimated and observed variance, has become a major goal for research on hypertension. Here we provide evidence that epigenetic alterations may contribute importantly to this problem. New technologies that enable the rigorous assessment of epigenetic changes and its phenotypic consequences may provide the basis for explaining the missing heritability of arterial hypertension and offer new possibilities for treatment and/or prevention.

\section{Acknowledgements}

None.

\section{Financial support and sponsorship}

This work was supported by the Swiss National Science Foundation, the Swiss Society of Hypertension, the Swiss Society of Cardiology, and the Placide Nicod Foundation. 


\section{Conflicts of interest}

There are no conflicts of interest.

\section{REFERENCES AND RECOMMENDED READING}

Papers of particular interest, published within the annual period of review, have been highlighted as:

- of special interest

- of outstanding interest

1. Cowley AW Jr, Nadeau JH, Baccarelli A, et al. Report of the National Heart, Lung, and Blood Institute Working Group on epigenetics and hypertension. Hypertension 2012; 59:899-905.

2. Stephan S, Lim EA. A comparative risk assessment of burden of disease and injury attributable to 67 risk factors and risk factor clusters in 21 regions, 1990-2010: a systematic analysis for the Global Burden of Disease Study. Lancet 2012; 380:2224-2260.

3. Frazer KA, Murray SS, Schork NJ, Topol EJ. Human genetic variation and its contribution to complex traits. Nat Rev Genet 2009; 10:241-251.

4. Havlik RJ, Garrison RJ, Feinleib M, et al. Blood pressure aggregation in families. Am J Epidemiol 1979; 110:304-312.

5. Manolio TA, Collins FS, Cox NJ, et al. Finding the missing heritability of complex diseases. Nature 2009; 461:747-753.

6. Zuk O, Hechter E, Sunyaev SR, Lander ES. The mystery of missing heritability: genetic interactions create phantom heritability. Proc Natl Acad Sci U S A 2012; 109:1193-1198.

7. Millis RM. Epigenetics and hypertension. Curr Hypertens Rep 2011; 13: $21-28$.

8. Rexhaj E, Paoloni-Giacobino A, Rimoldi SF, et al. Mice generated by in vitro fertilization exhibit vascular dysfunction and shortened life span. J Clin Invest 2013; 123:5052-5060.

9. Barker DJ. The fetal and infant origins of disease. Eur J Clin Invest 1995; 25:457-463.

10. Ost A, Lempradl A, Casas $E$, et al. Paternal diet defines offspring chromatin state and intergenerational obesity. Cell 2014; 159:1352-1364.

11. Roseboom TJ, van der Meulen $\mathrm{JH}$, Osmond $\mathrm{C}$, et al. Coronary heart disease after prenatal exposure to the Dutch famine. Heart 2000; 84:595-598.

12. Van Abeelen AF, Veenendaal MV, Painter RC, et al. The fetal origins of hypertension: a systematic review and meta-analysis of the evidence from animal experiments of maternal undernutrition. J Hypertens 2012; 30:22552267.

13. Baccarelli A, Rienstra M, Benjamin EJ. Cardiovascular epigenetics: basic concepts and results from animal and human studies. Circ Cardiovasc Genet 2010; 3:567-573.

14. Yazbek SN, Spiezio SH, Nadeau JH, Buchner DA. Ancestral paternal genotype controls body weight and food intake for multiple generations. Hum Mol Genet 2010; 19:4134-4144.

15. Lane M, Robker RL, Robertson SA. Parenting from before conception.

Science 2014; 345:756-760.

This review points out the importance of epigenetics as one of the most relevant mechanisms involved in fetal programming of adult diseases.
16. Kamphuis $E$, van Wely $M$, Repping $S$, et al. Should the individual preterm birth risk be incorporated into the embryo transfer policy in in vitro fertilisation? A decision analysis. BJOG 2015; 122:825-833.

17. Rimoldi SF, Sartori C, Rexhaj E, et al. Antioxidants improve vascular function in children conceived by assisted reproductive technologies: a randomized double-blind placebo-controlled trial. Eur J Prev Cardiol 2014. [Epub ahead of print]

18. Rimoldi SF, Sartori C, Rexhaj E, et al. Vascular dysfunction in children conceived by assisted reproductive technologies: underlying mechanisms and future implications. Swiss Med Wkly 2014; 144:w13973.

19. Scherrer U, Rimoldi SF, Rexhaj E, et al. Systemic and pulmonary vascular dysfunction in children conceived by assisted reproductive technologies. Circulation 2012; 125:1890-1896.

20. Von Arx R, Allemann $Y$, Sartori C, et al. Right ventricular dysfunction in children and adolescents conceived by assisted reproductive technologies. J Appl Physiol . (in press) doi:10.1152/japplphysiol.00533.2014.

21. Ceelen $M$, van Weissenbruch $M M$, Vermeiden JP, et al. Cardiometabolic differences in children born after in vitro fertilization: follow-up study. J Clin Endocrinol Metab 2008; 93:1682-1688.

22. Rexhaj $E$, Von Arx R, Cerny D, et al. Assisted reproductive technologiesinduced premature vascular ageing persists and evolves into arterial hypertension in adolescents. FASEB J 2015; 29:957.9.

23. Chen $X$, Wang $Y$. Tracking of blood pressure from childhood to adulthood: a systematic review and meta-regression analysis. Circulation 2008; 117: 3171-3180.

24. Ferrannini E, Buzzigoli G, Bonadonna R, et al. Insulin resistance in essential hypertension. N Engl J Med 1987; 317:350-357.

25. Cook S, Scherrer U. Insulin resistance, a new target for nitric oxide-delivery drugs. Fundam Clin Pharmacol 2002; 16:441-453.

26. Scherrer $U$, Randin $D$, Vollenweider $P$, et al. Nitric oxide release accounts for insulin's vascular effects in humans. J Clin Invest 1994; 94:2511-2515.

27. Scherrer $U$, Sartori $C$. Insulin as a vascular and sympathoexcitatory hormone: implications for blood pressure regulation, insulin sensitivity, and cardiovascular morbidity. Circulation 1997; 96:4104-4113.

28. Scherrer U, Rexhaj $E$, Allemann $Y$, et al. Cardiovascular dysfunction in children - conceived by assisted reproductive technologies. Eur Heart J . (in press).

This article outlines in a comprehensive manner the most relevant studies in fetal programming of premature vascular ageing induced by assisted reproductive technologies.

29. Lewandowski AJ, Leeson P. Preeclampsia, prematurity and cardiovascular health in adult life. Early Human Develop 2014; 90:725-729.

30. Langley-Evans SC. Developmental programming of health and disease. Proc Nutr Soc 2006; 65:97-105.

31. Jayet PY, Rimoldi SF, Stuber T, et al. Pulmonary and systemic vascular dysfunction in young offspring of mothers with preeclampsia. Circulation $2010 ; 122: 488-494$.

32. Lazdam $M$, de la Horra $A$, Pitcher $A$, et al. Elevated blood pressure in offspring born premature to hypertensive pregnancy: is endothelial dysfunction the underlying vascular mechanism? Hypertension 2010; 56:159-165.

33. Kajantie E, Eriksson JG, Osmond C, et al. Preeclampsia is associated with increased risk of stroke in the adult offspring: the Helsinki birth cohort study. Stroke 2009; 40:1176-1180.

34. Rexhaj $\mathrm{E}$, Bloch J, Jayet PY, et al. Fetal programming of pulmonary vascular dysfunction in mice: role of epigenetic mechanisms. Am J Physiol Heart Circ Physiol 2011; 301:H247-H252. 